OPEN ACCESS

Edited by:

Shoichiro Kurata,

Tohoku University, Japan

Reviewed by:

Mika Rämet,

University of Tampere, Finland

Osamu Takeuchi,

Kyoto University, Japan

*Correspondence:

Dominique Ferrandon

D.Ferrandon@ibmc-cnrs.unistra.fr

Specialty section:

This article was submitted to Comparative Immunology,

a section of the journal

Frontiers in Immunology

Received: 24 July 2019

Accepted: 29 December 2020

Published: 05 March 2021

Citation:

Huang C, Xu R, Liégeois S, Chen D,

LiZ and Ferrandon D (2021) Differential

Requirements for Mediator Complex

Subunits in Drosophila melanogaster

Host Defense Against Fungal and

Bacterial Pathogens.

Front. Immunol. 11:478958.

doi: 10.3389/fimmu.2020.478958

\section{Differential Requirements for Mediator Complex Subunits in Drosophila melanogaster Host Defense Against Fungal and Bacterial Pathogens}

\author{
Chuqin Huang ${ }^{1}$, Rui Xu ${ }^{1,2}$, Samuel Liégeois ${ }^{1,2}$, Di Chen ${ }^{1}, \mathrm{Zi} \mathrm{Li}^{1}$ \\ and Dominique Ferrandon ${ }^{1,2 *}$
}

1 Sino-French Hoffman Institute, Guangzhou Medical University, Guangzhou, China, 2 Université de Strasbourg, UPR 9022 du CNRS, Strasbourg, France

The humoral immune response to bacterial or fungal infections in Drosophila relies largely on a transcriptional response mediated by the Toll and Immune deficiency NF- $\mathrm{KB}$ pathways. Antimicrobial peptides are potent effectors of these pathways and allow the organism to attack invading pathogens. Dorsal-related Immune Factor (DIF), a transcription factor regulated by the Toll pathway, is required in the host defense against fungal and some Gram-positive bacterial infections. The Mediator complex is involved in the initiation of transcription of most RNA polymerase B (PolB)-dependent genes by forming a functional bridge between transcription factors bound to enhancer regions and the gene promoter region and then recruiting the PolB pre-initiation complex. Mediator is formed by several modules that each comprises several subunits. The Med17 subunit of the head module of Mediator has been shown to be required for the expression of Drosomycin, which encodes a potent antifungal peptide, by binding to DIF. Thus, Mediator is expected to mediate the host defense against pathogens controlled by the Toll pathway-dependent innate immune response. Here, we first focus on the Med31 subunit of the middle module of Mediator and find that it is required in host defense against Aspergillus fumigatus, Enterococcus faecalis, and injected but not topically-applied Metarhizium robertsii. Thus, host defense against $M$. robertsii requires Dif but not necessarily Med31 in the two distinct infection models. The induction of some Tollpathway-dependent genes is decreased after a challenge of Med31 RNAi-silenced flies with either $A$. fumigatus or $E$. faecalis, while these flies exhibit normal phagocytosis and melanization. We have further tested most Mediator subunits using RNAi by monitoring their survival after challenges to several other microbial infections known to be fought off through DIF. We report that the host defense against specific pathogens involves a distinct set of Mediator subunits with only one subunit for C. glabrata or Erwinia carotovora carotovora, at least one for $M$. robertsii or a somewhat extended repertoire for $A$. fumigatus (at least eight subunits) and E. faecalis (eight subunits), with two subunits, 


\section{Med6 and Med11 being required only against A. fumigatus. Med31 but not Med17 is required in fighting off injected $M$. robertsii conidia. Thus, the involvement of Mediator in Drosophila innate immunity is more complex than expected.}

\section{Keywords: mediator complex, Drosophila innate immunity, Toll pathway, host defense against fungi and bacteria,} RNA interference, survival to infection, humoral immune response

\section{INTRODUCTION}

Fungal invasions represent one of the most difficult infectious diseases to cure nowadays, causing worldwide more than 1.6 million deaths per year. People are constantly exposed to fungi, which are controlled in a first line of defense by the innate immune system through the phagocytosis of inhaled spores by macrophages and especially through neutrophils. Nevertheless, fungi can cause diseases such as airway allergy, bronchitis in healthy patients while it may cause deadly invasive infections in immunocompromised patients $(1,2)$.

The simpler immune system of the genetic model organism Drosophila melanogaster makes the analysis of host-pathogen interactions during infections easier to investigate as it lacks the adaptive immunity arm found in higher vertebrates. The host defense against pathogens in Drosophila mainly encompasses three major arms: melanization, the cellular response (essentially phagocytosis), and the humoral immune responses (Toll and Immune deficiency (IMD) NF- $\kappa \mathrm{B}$ pathways). The IMD pathway is required to fight off Gram-negative bacterial infections whereas the Drosophila defenses against some Gram-positive bacteria and fungi mostly rely on the Toll pathway. Following the sensing of cell wall compounds or of the proteolytic activity of secreted microbial virulence factors in the hemolymph by circulating receptors, host proteolytic cascades will lead to the production of a mature Toll ligand, which will trigger an intracellular signaling pathway that activates the NF- $\mathrm{BB}$-like transcription factors Dorsal or Dorsal immune-related factor (DIF) (3-5). DIF mediates Toll pathway function in innate immunity in adult flies while it is redundant with Dorsal in larvae (6-8). The Toll pathway regulates the expression of tens of genes, including those encoding antimicrobial peptides such as Drosomycin as well as those coding for the less characterized Drosophila-induced Immune Molecules (DIMs)/Bomanins, effectors that may act in conjunction with as yet unidentified cofactors (9-12)

Aspergillus fumigatus is the fungus that was initially used to demonstrate that Toll pathway mutants are sensitive to fungal infections (13). Given its medical relevance, we have implemented an unbiased genetic screen in which we monitor the survival of mutant Drosophila lines to injected conidia of this fungus. To this end, we are using transgenic lines that express miRNAs designed to target specific genes under the control of a Gal4 driver expressed ubiquitously (14), as well as other RNAi lines (15). To bypass the developmental lethality potentially caused by the down-regulated expression of the targeted gene, the RNAi transgene is expressed only at the adult stage using the Gal80 thermosensitive system (16). In the screen, we found that flies knocked down by RNAi targeting either of two Mediator complex subunit genes (Med8, Med 31) by RNAi transgene at the adult stage succumb to $A$. fumigatus infection.

The Mediator complex, evolutionarily conserved from yeast to plants, invertebrates, and mammals, consists of a multiprotein complex (25 subunits in yeast and 33 subunits in mammals) which plays an essential role for the transcription of almost all genes transcribed by RNA polymerase B (Pol B) (17-19). The Mediator complex is composed of a central module and a CDK8 kinase module (CKM). The central module consists of three complexes of distinct subunits known as the head, the middle, which together form the core module, and the tail parts. The central module associates with the CKM, which contains four subunits, and co-activates the transcription of target genes, yet does not appear to be fully essential for Mediator function. The Mediator core complex serves as a functional bridge connecting a variety of transcription activators bound to enhancer regions to the transcriptional machinery at the basal promoter, which includes Pol B and general transcription factors, to initiate gene transcription. The first step of gene transcription is the binding of transcription factors to the enhancer regions. Then, there is a subsequent recruitment of the Mediator complex to the enhancer regions by its interaction(s) with transcription factors through the tail and middle modules. Finally, general transcription factors and Pol B are recruited through the core module to form the preinitiation complex on the core promoter of the target gene (20). Gene transcription is then initiated, a process facilitated by the Mediator complex. In fact, Mediator complex can contact hundreds of transcription activators through its different subunits, generally the tail ones, and transduces the signals from specific transcription factors under different conditions (21). All subunits of the Mediator complex are recruited to the enhancer region; the CDK8 kinase module is transiently dissociated from the complex during the interaction with core promoters, however, its exact role is still not clear (20). Besides its role in the initiation of transcription, other studies have revealed that the Mediator complex also play roles at some other stages of transcription such as elongation, termination, processing of mRNA, and in epigenetic regulation as well as noncoding RNA activation (22).

Even though Mediator is required for the transcription of nearly all Pol B transcripts, half of its subunits appear to have specific functions. In Drosophila, most studies have focused on the role of the Mediator complex during development, e.g (23, 24). A previous study revealed that one of the Drosophila Mediator complex subunits, dTRAP80 (a homologue of the head module component Med17), is required for DIFdependent transcriptional activation of the Drosomycin gene in 
cultured cells and in vitro-translated Med17 has been shown to physically interact with both Dorsal and DIF by GST pull down (25). As stated above, two independent hits of our genetic screen are Med8 and Med31. Med31 but not Med8 may bind to DIF and Dorsal and both have been reported to bind to Med17 in a largescale effort to map protein-protein interactions in Drosophila by coaffinity purification of protein complexes (26). Whereas Med17 and Med8 both belong to the head module, Med31 belongs to the middle module, yet interacts, possibly indirectly, with several head module subunits besides Med17: Med6, Med11, Med18, and Med20 of the head module, and also Med7 from the middle module and Med14. Med8 appears to associate with subunits from all three central modules, including the Med14 scaffolding subunit, Med6, Med11, Med 17, and Med18 of the head module, Med4, Med7, Med10 of the middle module and Med15, Med16, Med23, Med25, Med27, and Med30 of the tail module (27). We therefore hypothesized that Med31, and possibly Med8, may play a role in Drosophila host defense against $A$. fumigatus infection through the facilitation of transcriptional activation mediated by DIF. We have extensively characterized the Med31 A. fumigatus susceptibility phenotype and also investigated the function of Med31 in host defense against other pathogens including the dimorphic or monomorphic yeasts Candida albicans or Candida glabrata, the entomopathogenic fungus Metarhizium robertsii, the Gram-positive bacterium Enterococcus faecalis and the Gramnegative bacterium Erwinia carotovora carotovora 15 (Ecc15). The Toll pathway is required for host defense against all these pathogens, except the last one. We further studied the role of most other identified Mediator subunits in Drosophila host defense, with a special emphasis on Med17, the subunit reported to directly bind to DIF. Our results delineate an unexpectedly complex picture of the Mediator complex in host defense that does not fit with it acting solely through the DIF transcription factor.

\section{MATERIALS AND METHODS}

\section{Fly Strains and Husbandry}

Flies were raised at $25^{\circ} \mathrm{C}, 60 \%$ humidity with $12 \mathrm{~h}$ of light/dark cycle. The flies were fed with a semi-solid medium which consists of $7.8 \% \mathrm{w} / \mathrm{v}$ of corn flour, $6.3 \% \mathrm{w} / \mathrm{v}$ of glucose, $3.2 \%$ $\mathrm{w} / \mathrm{v}$ of yeast dry powder, $0.9 \% \mathrm{w} / \mathrm{v}$ of agar, $0.2 \% \mathrm{w} / \mathrm{v}$ of sorbitol (except for A. fumigatus infected flies because A. fumigatus is sensitive to the sorbitol preservative), $0.07 \% \mathrm{w} / \mathrm{v}$ of $\mathrm{CaCl} 2,3.2 \%$ $\mathrm{w} / \mathrm{v}$ of sucrose, $0.15 \% \mathrm{w} / \mathrm{v}$ of $\mathrm{p}$-Hydroxybenzoic Acid Methyl Ester and water.

The TRiP RNAi lines were obtained from the TsingHua Fly Center (THFC), the GD RNAi lines come from the Shanghai Institute for Biological Sciences (originally from the Vienna Drosophila Research Center, Austria). The insert was checked by sequencing for each line. UAS-mCherry-sh (BDSC_35785) and VDRC_60000 were used as controls for TRiP lines and GD lines, respectively. Supplementary Table 1 lists the RNAi lines used in this study. Males from the RNAi lines or their controls were crossed with Ubi-Gal4, tub-Gal80 ${ }^{\text {ts }}$ virgins. Crosses were set-up at $25^{\circ} \mathrm{C}$ for three days to ensure an efficient fertilization of females by males. Adults were then transferred to another tube while the tube containing eggs was moved to $18^{\circ} \mathrm{C}$, in order to keep the inhibition of Gal4 by $\mathrm{Gal}^{\mathrm{ts}}$ and bypass developmental lethality.

Soon after the $\mathrm{F} 1$ progeny hatched, flies were shifted at $29^{\circ} \mathrm{C}$ to inhibit $\mathrm{Gal}^{\mathrm{ts}}{ }^{\mathrm{ts}}$ and activate $\mathrm{Gal} 4$ to initiate the transgene expression. Flies were kept at $29^{\circ} \mathrm{C}$ for 5 days to ensure the downregulation of the genes of interest prior to immune challenge.

\section{Microbial Strains and Growth Conditions}

The RFP-labeled wild-type Aspergillus fumigatus strain was a kind gift from Drs. Anne Beauvais and Jean-Paul Latge (Institut Pasteur, Paris). A. fumigatus was cultured on potato dextrose agar (PDA) medium plates supplemented with $0.1 \mathrm{~g} / \mathrm{l}$ chloramphenicol (Huankai Microbio Tech, China) in a tissue culture incubator under $5 \% \mathrm{CO}_{2}$ at $29^{\circ} \mathrm{C}$. Metarhizium robertsii (ARSEF 2575) was grown on PDA plates from BD Company, USA (\#213400) at $25^{\circ} \mathrm{C}$ for 7 to 14 days. Of note, we did not use the same PDA plates for A. fumigatus and $M$. robertsii because the PDA used for A. fumigatus contains chloramphenicol, which affects the growth of $M$. robertsii.

Candida albicans (CAF 2.1) and Candida glabrata (28) were cultured on Yeast extract Peptone Dextrose (YPD) Agar plates for two days at $29^{\circ} \mathrm{C}$, from which one colony was plated on a new plate again for two days at $29^{\circ} \mathrm{C}$, and then this plate was kept at $25^{\circ} \mathrm{C}$ for infections for four weeks.

E. faecalis (OG1RF) and the Pectobacterium carotovorum strain Erwinia carotovora carotovora (Ecc15) were cultured in Luria-Bertani (LB) agar plates overnight at $37^{\circ} \mathrm{C}$. A single colony was inoculated in $\mathrm{LB}$ liquid medium at $37^{\circ} \mathrm{C}$ and overnight shaking.

\section{Microbial Preparation and Infection Experiments}

Prior to A. fumigatus injection or $M$. robertsii natural infection, flies were raised on $100 \mathrm{mM}$ sucrose for two days to eliminate sorbitol (an antifungal compound present in the fly food) from the flies. Prior to $M$. robertsii injection, flies were raised on regular food. $M$. robertsii and A. fumigatus conidia were collected from the surface of the PDA plate by adding three $\mathrm{ml}$ of either PBST (PBS containing $0.01 \%$ Tween-20) for injections, or sterilized deionized demineralized water containing $0.01 \%$ Tween-20 for natural infections. The concentration of the conidia was counted using a hemocytometer and then adjusted to the adequate working concentration. The working concentrations were $5 \times 10^{7}$ A. fumigatus conidia/ml and $1 \times 10^{7}$ M. robertsii conidia/ml in PBST for injections, and $5 \times 10^{4}$ conidia/ml in water containing $0.01 \%$ Tween-20 for $M$. robertsii natural infection.

For $M$. robertsii natural infection, anesthetized flies were incubated into the conidia solution and shaken for $30 \mathrm{~s}$, before being dried on Millipore AP1003700 filter paper adapted to a vacuum pump. $M$. robertsii-naturally infected flies were then raised on a vial containing a filter paper with $100 \mathrm{mM}$ sucrose. $A$. 
fumigatus-injected flies were kept on food without sorbitol whereas $M$. robertsii-injected flies were fed regular food.

Overnight cultures of E. faecalis and Ecc15 were centrifuged at $3,500 \mathrm{~g}, 4^{\circ} \mathrm{C}$ for $10 \mathrm{~min}$. The pellet was washed twice in PBS. E. faecalis and Ecc15 were prepared in PBS at working concentrations of OD600 $=0.1$ and 1 , respectively.

Injection of $A$. fumigatus, M. robertsii, E. faecalis or Ecc15 was performed by injecting $4.6 \mathrm{~nL}$ of working solutions, or the same volume of PBS as a control, into flies by using a microinjector (Nanoject, Drummond) and appropriate capillaries. Infection of C. albicans or C. glabrata was performed by pricking through a sharpened tungsten needle dipped into a single colony directly taken on plates (28).

\section{Survival Assays}

The survival assays were performed with 15-25 females per tube, in triplicates. Infected flies were incubated at $29^{\circ} \mathrm{C}$ with $60 \%$ humidity. Log-Rank statistical tests were performed with GraphPad Prism 6. Experiments were performed at least twice, except for the following experiments that were performed only once: $M$. robertsii natural infections: Med6, Med8, Med11, Med22, Med24; C. glabrata infections: Med12, Med14, Med21; E. faecalis infections: Med11, Med12; Ecc15 infections: Med11, Med12.

\section{Detection of AMP Expression Level After Infection}

RNA was extracted from four flies per sample in triplicates or quadruplicates. Flies were homogenized with $1 \mathrm{ml}$ of Trizol reagent (Thermo Fisher Scientific, \#15596018) in a microfuge tube using a pestle and the RNA extraction was performed according to the manufacturer instructions. Two hundred $\mu \mathrm{L}$ of chloroform was added to the samples, which were vortexed and incubated for $5 \mathrm{~min}$ at room temperature. Then, samples were centrifuged at $13,000 \mathrm{~g}$ at $4^{\circ} \mathrm{C}$ for $10 \mathrm{~min}$. The water phase at the top of the samples was collected and mixed with the same volume of isopropanol and vortexed again. Samples were centrifuged at $13,000 \mathrm{~g}$ at $4^{\circ} \mathrm{C}$ for $10 \mathrm{~min}$. The pellet was washed with a solution of $75 \%$ ethanol in water and air dried. Then, RNAs were re-suspended in $35 \mu$ l of RNase-free water. The quality and the concentration of the total RNA were measured using a Nanodrop 2000 (Thermo Fisher Scientific). The reverse transcription of $800 \mathrm{ng}$ of total RNA was made with a cDNA synthesis kit (TransGene Biotech, \#AT-341), Quantification of the target gene expression level was performed by quantitative Polymerase Chain Reaction (qPCR) with the SYBR Green Supermix kit (Vazyme Biotech, Q311-02). The relative gene expression was normalized to the expression level of the housekeeping gene Rpl32, which encodes ribosomal protein 49. Digital PCR was performed on $\mathrm{cDNAs}$ at $1 \mathrm{ng} / \mu \mathrm{L}$ as described (29). The list of primers used for this study is found in Table S2.

Wild-type flies pricked with concentrated cultures of Micrococcus luteus were used as positive controls for Drosomycin and DIM1, or Escherichia coli for Diptericin expression (30). The $\Delta \Delta \mathrm{Ct}$ method was used to normalize the values as following: we gave a value of 1 for the expression level obtained for Drosomycin and DIM1 $24 \mathrm{~h}$ after infection with M. luteus and for the expression level obtained for Diptericin $6 \mathrm{~h}$ after infection with E. coli.

\section{Western Blot}

Four hours after a challenge with $M$. luteus, the hemolymph from 20 flies was collected into $40 \mu \mathrm{l}$ of PBS containing Protease Inhibitor Cocktail (Thermo Fisher Scientific) by centrifugation $\left(3,500 \mathrm{~g}, 30 \mathrm{~min}, 4^{\circ} \mathrm{C}\right)$ after cutting the tip of the abdomen (28). The protein concentration of the sample was measured by using a BCA kit (Beyotime Biotechnology). Samples were mixed with SDS-PAGE loading buffer (Beyotime Biotechnology) and boiled for $5 \mathrm{~min}$. A SDS-PAGE electrophoresis gel was performed using $20 \mu \mathrm{g}$ of protein. Proteins were transferred to a polyvinylidene fluoride (PVDF) membrane $(0.22 \mu \mathrm{m})$.

The nitrocellulose membrane with transferred proteins was blocked in PBST with 5\% BSA at room temperature for $1 \mathrm{~h}$. The membranes were incubated in a 1:10,000 rabbit anti-PPO1 primary antibody solution (a kind gift from Erjun Ling, Shanghai) in PBST with BSA overnight at $4^{\circ} \mathrm{C}$ (31). The membranes were washed and incubated in a secondary antirabbit HRP antibody (1:10000) for $1 \mathrm{~h}$ at room temperature.

\section{Phagocytosis Assay}

Three to seven days old adult females were injected with $69 \mathrm{~nL}$ of latex beads (Thermo Fisher Scientific), 16\% w/v (re-suspended in PBS and sonicated prior injection). Control flies were injected with the same volume of PBS. Twenty-four hours post-injection, flies were injected with $69 \mathrm{~nL}$ pHrodo ${ }^{\mathrm{TM}}$ Red E. coli BioParticles ${ }^{\mathrm{TM}}$ Conjugate (Thermo Fisher Scientific, P35361). The phagocytic activity was observed under a fluorescence microscope (Zeiss Imager.M2) after $30 \mathrm{~min}$. The red fluorescence was quantified in fields of same size. Ten flies of each line were scored in each experiment and three independent experiments were performed.

\section{Microbial Load Counts}

A. fumigatus and C. glabrata microbial loads after infection were counted after plating a homogenate of single whole flies. Each single fly was transferred into a $1.5 \mathrm{ml}$ Eppendorf tube containing $50 \mu \mathrm{l}$ of PBST and smashed by a tissue homogenizer. After a few seconds of centrifugation, the entire homogenate product was plated on PDA, and incubated at $29^{\circ} \mathrm{C}$. Colony forming units (CFUs) were counted after 2-3 days of incubation. In the case of C. glabrata, a 1:100 or 1:1,000 dilution of the homogenate product was plated depending on the time after infection.

E. faecalis loads were counted on hemolymph collected from single flies. A series of dilution was performed from 1 to $1: 10^{8}$ in PBS. Ten $\mu$ of each dilution was plated in duplicate on LB agar and incubated at $37^{\circ} \mathrm{C}$. Colony forming units (CFUs) were counted the next day.

\section{Statistical Analysis}

All statistical analyses were performed by using GraphPad Prism 7. The Mann-Whitney or Kruskall-Wallis tests was used for the statistical analysis of all the data except survival experiments. Survival curves were plotted and analyzed by Log-Rank test (Kaplan-Meier method). 


\section{RESULTS}

\section{Med31 RNAi Flies are Susceptible to Injected Aspergillus fumigatus Conidia}

We have established that as little as five A. fumigatus conidia injected on average per fly are sufficient to kill MyD88immunodeficient flies and are routinely injecting 250 conidia per fly (Xu et al., in preparation). As shown in Figure 1A, this dose rapidly kills $M y D 88$ whereas only a moderate proportion of $m$ Cherry RNAi control flies succumbed to this challenge in most experiments. Med31 RNAi flies displayed a high sensitivity to injected A. fumigatus in ten independent experiments, which was however not as pronounced as for $M y D 88$ flies. We confirmed this result using two other independent RNAi lines and also checked by classical (not shown) and digital RT-qPCR that all three RNAi lines effectively decreased the steady-state levels of MED31 transcripts (Figures S1A-C). Next, we measured the fungal load to determine whether it increases in the mutant background, as has been reported for immuno-deficient flies challenged with pathogens $(28,32)$. At $24 \mathrm{~h}$ after infection, the titer was somewhat higher in MyD88 and Med31 RNAi flies than in $m$ Cherry RNAi control flies (Figure 1B). However, the fungal burden did not increase at $48 \mathrm{~h}$ (Figure 1C). We next monitored the induction of the Toll pathway using the expression level of two of its target genes, Drosomycin and BomS1/DIM1 (33). The injection of 250 A. fumigatus conidia mildly induced the expression of these two genes, which was reduced in Med31 RNAi flies (Figures 1D, E). Thus, Med31 is required for the full

A

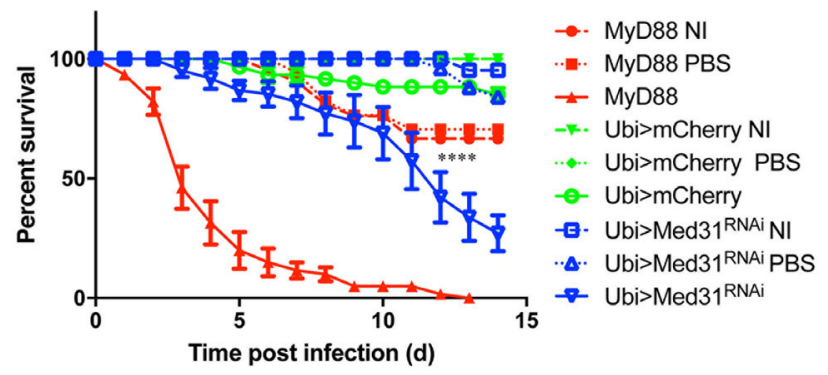

B
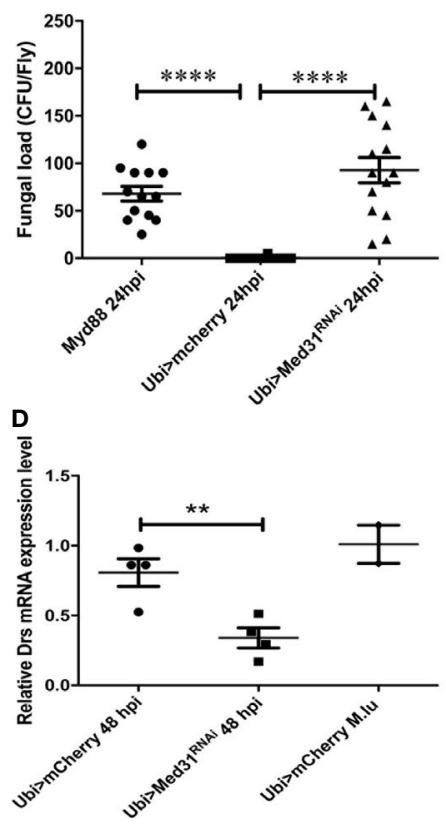

C

E

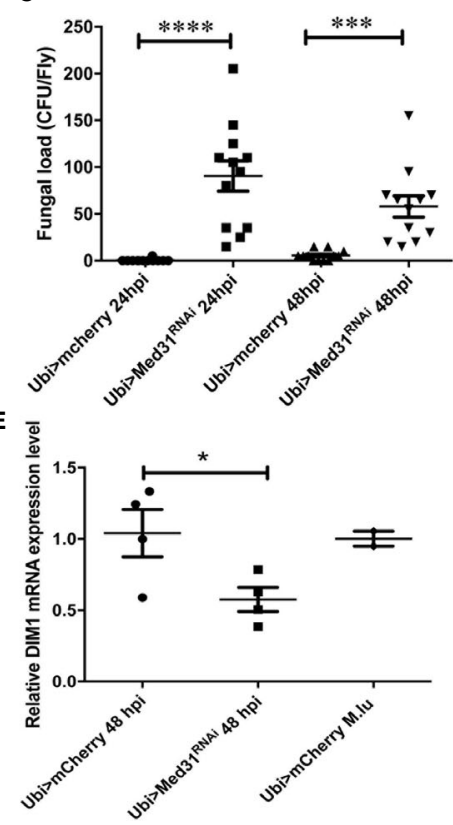

FIGURE 1 Med31 RNA flies are susceptible to A. fumigatus infection. (A) Survival of Drosophila after A. fumigatus infection. MyD88 is the positive control line (red), Ubi>mCherry RNAi line is the wild type control line (green) and Ubi>Med31 RNAi line is the experimental line (blue). Each infected line has a non-infected (NI, dashed lines) and PBS-injected control (dotted lines). Med31 RNAi flies succumbed faster to infection than the wild type controls. Infected Med31 RNAi flies vs. infected $m$ Cherry RNAi flies: log-rank test, ${ }^{\star \star \star \star} \mathrm{P}<0.0001$. The survival curves are representative of 10 independent experiments. (B, C) Fungal load of whole single flies after 24 and $48 \mathrm{~h}$ of infection. Each dot represents a single fly. The fungal load was higher in MyD88 and Med31 RNAi flies than in control flies at $24 \mathrm{~h}$ post infection. It did not increase in Med31 RNAi flies at $48 \mathrm{~h}$ post infection compared to $24 \mathrm{~h} .{ }^{* \star \star} \mathrm{P}<0.0001 .{ }^{* \star *} \mathrm{P}<0.001$. (D, E) Expression levels of Drosomycin (D) and DIM1 (E) at $48 \mathrm{~h}$ post infection, normalized to Rp/32 (RP49 protein coding gene) housekeeping gene expression. The expression of control flies challenged with the nonpathogenic Gram-positive bacterium Micrococcus luteus is given for reference. Each dot represents one sample containing four flies. Med31 RNAi flies displayed decreased Drosomycin and DIM1 expression levels at $48 \mathrm{~h}$ post infection. ${ }^{*} \mathrm{P}<0.05$. Mean $\pm \mathrm{SEM}$ are indicated (B-E). 
transcriptional induction of Drosomycin, likely by recruiting Pol B to the Drosomycin and DIM1 promoters bound by DIF.

\section{Med31 RNAi Flies Display a Moderate Sensitivity to Enterococcus faecalis Infection}

When challenged with E. faecalis, Med31 RNAi flies displayed a sensitivity to this infection that was intermediate between those of $M y D 88$ and wild-type control flies in seven out of nine experiments (Figure 2A), whereas they behaved almost like wild-type flies in the two other experiments. To corroborate these results, we measured the bacterial load and found that it was increased on average 32-fold in the Med31 RNAi flies with respect to $m$ Cherry RNAi control flies (Figure 2B). Unexpectedly, we did not find a significant decrease in Drosomycin induction by E. faecalis challenge in three independent experiments, although the mean induction of Drosomycin was somewhat decreased when compared to controls (Figure 2C); in a digital RTqPCR experiment, we indeed found a significant difference. However, there was a significant difference when monitoring another read-out of Toll pathway activation, BomS1/DIM1 transcript levels (Figure
2D), which was confirmed by digital RTqPCR. The reduction was however modest. Thus, Med31 RNAi flies appear to have reduced host defenses against two pathogens known to be effectively killing Toll pathway-deficient flies.

\section{Test of Med31 RNAi Flies in Further Infection Models}

The Toll pathway has been reported to play an essential role in host defense against entomopathogenic fungi such as Metarhizum robertsii and pathogenic yeasts such as Candida albicans and C. glabrata (4, 28, 34, 35). Entomopathogenic fungi invade the host body cavity upon the deposition of spores on the cuticle of the insect ("natural" infection model) or can be artificially directly injected inside the fly (septic injury model mimicking a wound). Interestingly, we found in nine "natural" infection experiments that Med31 RNAi flies behaved as wildtype control flies in this infection paradigm (Figure 3A) whereas they displayed a moderate but reproducible susceptibility to injected $M$. robertsii in five experiments (Figure 3B). With respect to pathogenic yeasts, Med31 RNAi flies displayed a weak susceptibility to C. albicans in two out of four experiments (Figure 3C) while it was not sensitive to C. glabrata in six out of nine
A

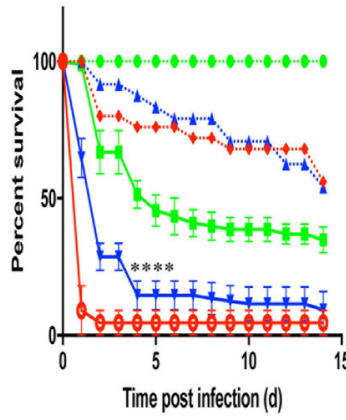

C

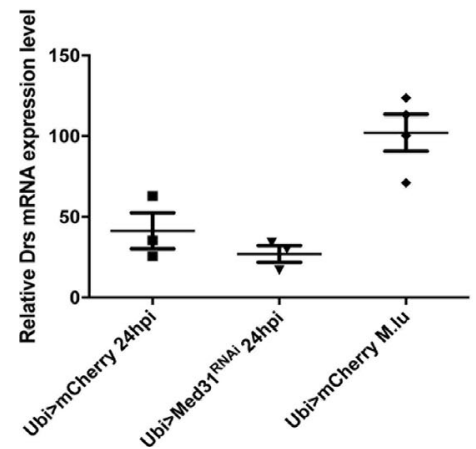
-4. MyD88 PBS

* MyD88

+ UbismCherry
B

*. UbismCherryPBS

-1. Ubi Med31 $1^{\mathrm{RNA}} \mathrm{PBS}$

+ UbipMed31 ${ }^{R N A}$

D
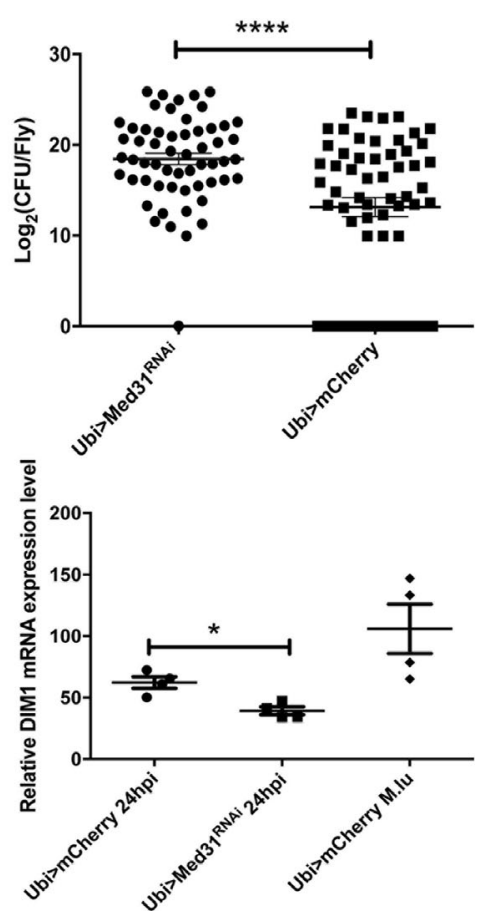

FIGURE 2 | Med31 RNAi flies are susceptible to E. faecalis infection. (A) Survival of Drosophila after E. faecalis infection. MyD88 was used as a positive control line (red), Ubi>mCherry RNAi line represented the wild type control line (green) and the Ubi >Med31 RNAi line survival is shown in blue. For each infected line a PBSinjected control was also performed (dotted lines). Med31 RNAi flies succumbed faster to infection than the wild type controls. Infected Med31 RNAi flies vs. infected mCherry RNAi flies: log-rank test, ${ }^{\star \star \star \star} \mathrm{P}<0.0001$. The survival curves are representative of nine independent experiments. (B) Bacterial load in the hemolymph after $24 \mathrm{~h}$ of infection. Each dot represents the burden of a single fly. The bacterial load in the Med31 RNAi flies was higher than the controls at $24 \mathrm{~h}$ post infection. ${ }^{\star \star \star} \mathrm{P}<0.001$. (C, D) Expression level of Drosomycin (C) and DIM1 (D) normalized to the Rp/32 house keeping gene $48 \mathrm{~h}$ post infection. Each dot represents one sample containing four flies. Septic infection with M. luteus was a positive control for Drosomycin and DIM1 expression. Drosomycin expression level in Med31 RNAi flies was not significantly different from control flies (C) but DIM1 expression level was significantly decreased at $24 \mathrm{~h}$ post infection. ${ }^{*} \mathrm{P}<0.05$. Mean \pm SEM are indicated (B-D). 
A

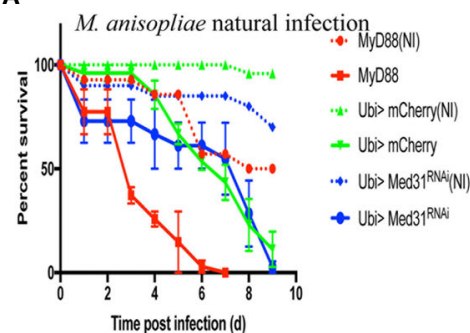

C

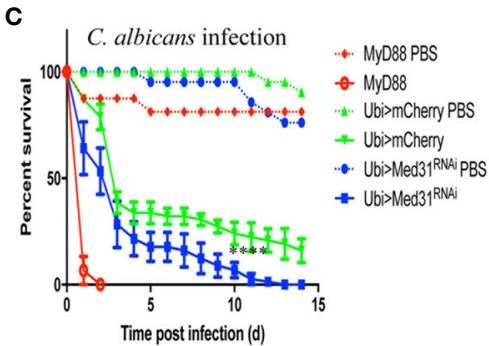

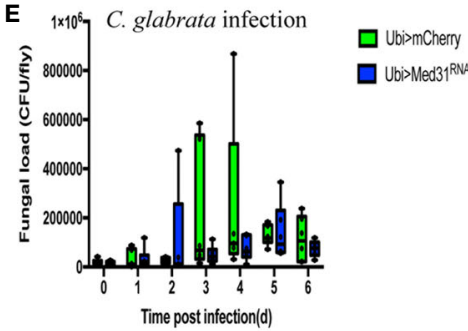

G

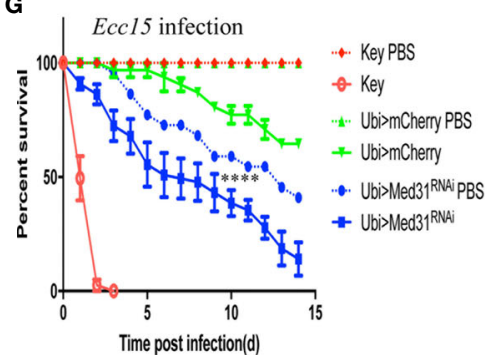

B
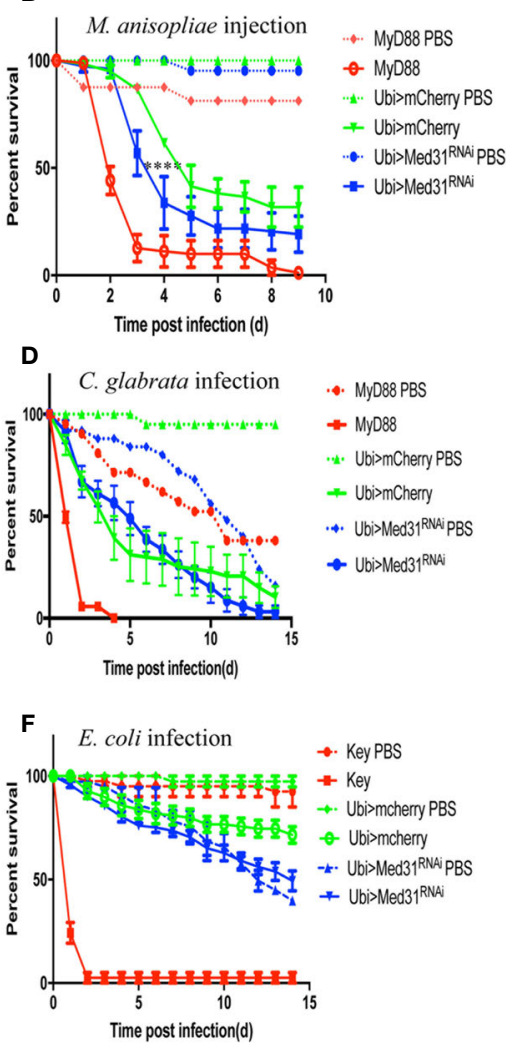

$\mathbf{H}$

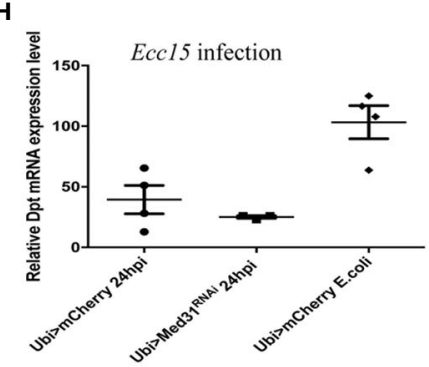

FIGURE 3 | Susceptibility of Med31 RNAi flies to other pathogens. Survival of Med31 RNAi flies challenged with other pathogens. MyD88 represents the positive control line (red) for flies infected with M. anisopliae, C. albicans, C. glabrata, and E. faecalis; kenny, a member of the IMD pathway, is the positive control line (red) for fly lines infected with Ecc15 or E. coli, Ubi>mCherry RNAi line corresponds to the wild type control line (green) and Ubi>Med31 RNAi line (blue). For each infected line a PBS-injected control was also performed (dotted lines). (A) Survival of Med31 RNAi flies after M. robertsii natural infection. The survival curve is representative of nine independent experiments. (B) Survival of Med31 RNAi flies following M. robertsii conidia injection. Infected Med31 RNAi flies vs. infected mCherry RNAi flies: log-rank test, ${ }^{\star \star \star \star} \mathrm{P}<0.0001$. The survival curves are representative of five independent experiments. (C) Survival of Med31 RNAi flies following C. albicans infection. Infected Med31 RNAi flies vs. infected mCherry RNAi flies: log-rank test, ${ }^{\star \star \star *} \mathrm{P}<0.001$. The survival curves are representative only of two out of four independent experiments, the other two not displaying any difference between Med31 RNAi and control flies. (D, E) Survival of Med31 RNAi flies and the fungal load following C. glabrata infection. The survival curves are representative of nine independent experiments. (F) Survival of Med31 RNAi flies following E. coli infection. The survival curves are representative of two independent experiments. (G) Survival of Med31 RNAi flies after Ecc15 challenge. The survival curves are representative of nine experiments. (H) Diptericin expression level normalized to the Rp/32 house keeping gene at $24 \mathrm{~h}$ post infection with Ecc15. Each dot represents one sample containing four flies. Septic infection with E.coli was a positive control for Diptericin expression. Mean \pm SEM are indicated, except for $3 E$ where the median is displayed.

survival experiments (Figure 3D). We found that the C. glabrata burden was not differing between mCherry RNAi and Med31 RNAi flies during the course of the infection (Figure 3E). Thus, Med31 does not appear to be required to the same extent in host defense against microbial infections depending on the pathogen, even though all of these microbes are controlled, at least to some degree, by the Toll pathway. We also checked whether Med31 affects the host defense against Gram-negative pathogens. The weakly pathogenic Escherichia coli did not kill Med31 RNAi flies more efficiently than a PBS-injection control in two independent 
experiments (Figure 3F). We found that Med31 RNAi flies were also insensitive to Ecc15 challenge in seven out of 11 experiments (Figure 3G). We also checked whether IMD pathway signaling was affected in Med31 RNAi flies by measuring the steady-state transcript levels of Diptericin. No significant difference was recorded (Figure 3H). In conclusion, the requirement for full Med31 function in host defense against bacterial or fungal infection varies according to the pathogen and the unique suite of host defenses engaged in each case. As Dif has been reported to be required in host defense against $M$. robertsii in the "natural infection" model, the lack of a requirement for Med31 in this infection is unexpected given its involvement in the host defense against $A$. fumigatus, $E$. faecalis, and injected $M$. robertsii conidia. We cannot however exclude the possibility that another Mediator subunit mediates an interaction with DIF in the other infections that are not modulated through Med31.

\section{Med31 Does Not Appear to be Required for the Melanization nor for the Cellular Immune Response}

We have tested whether Med31 plays a role in two other host defenses, melanization and phagocytosis. We did not notice any alteration of the melanin plug formed at the wounding site in Med31 RNAi flies. We further tested at the molecular level whether the proteolytic processing of prophenol oxidase into mature phenol oxidase was impaired in these flies, as this represents a key step in the melanization response. We found in four out of five experiments that prophenol oxidase was equally or better cleaved in the mutant flies as compared to wild-type controls whereas in one experiment a minor unprocessed form remained while the control was fully cleaved (Figure 4A). We conclude that Med31 does not influence melanization after a septic injury.

We next checked whether the basal phagocytic machinery was functional in Med31 RNAi flies by injecting $\mathrm{pH}$-rodo-labeled E. coli that emit red fluorescence when placed in an acidic environment such as that encountered in mature phagolysosomal vesicles. Figures $\mathbf{4 B}-\mathbf{C}$ show that the uptake of these particles by hemocytes located on the fly dorsal vessel was not dramatically altered when Med31 expression was ubiquitously knocked-down.

\section{A Mini-Screen to Identify Other Med Subunits Involved in Host Defense Against Aspergillus fumigatus}

To obtain a better understanding of the role of the Mediator complex in host defense in the Drosophila model, we decided to test the available RNAi lines targeting the genes encoding other subunits of this complex. A limitation of the RNAi approach is that the efficiency of interference may be varying. As the Mediator complex plays an essential role in development, we reasoned that expressing the RNAi transgene throughout development should severely alter the proportion of adult flies hatching from a cross between the RNAi line and a ubi-Gal4 driver line. This strategy should therefore allow us to validate the efficiency of the RNAi lines in blocking their targets. Indeed, we
A

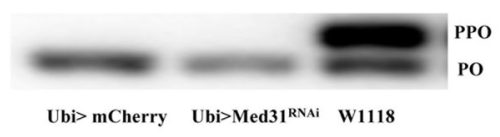

B

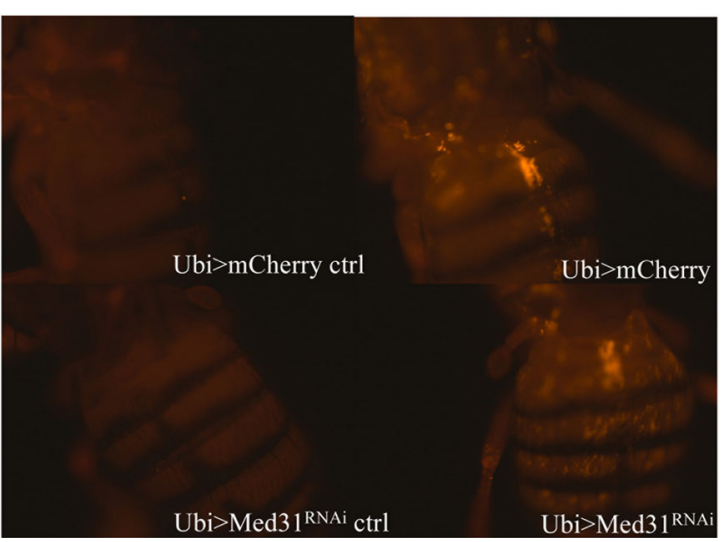

C

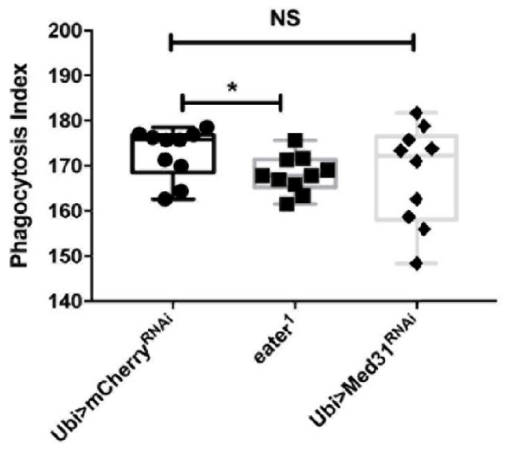

FIGURE 4 | Melanization and phagocytosis are not affected in Med31 RNAi flies. (A) prophenol oxidase (PPO) cleavage four hours after a M. luteus challenge was detected by Western blot analysis. The cleavage of PPO into phenol oxidase (PO) is shown in the picture. PPO cleavage into PO was total for both Med31 RNAi and mCherry RNAi control flies, while that induced in another wild-type control, w1118, was only partial. This blot is representative of three out of four independent experiments. The phagocytic activity of flies was detected using injected $\mathrm{pH}-\mathrm{RODO}$-labelled $E$. coli, which become fluorescent when internalized into mature phagosomes. (B) Flies were injected with latex beads as a phagocytosis-deficient control (left panels). The phagocytic activity of Med31 RNAi flies was not altered compared to the mCherry RNAi control flies (right panels). (C) Quantification of the fluorescence emitted by internalized bacteria. The eater ${ }^{1}$ phagocytosisdeficient mutant flies represent a positive control. There was no significant difference between Med31 RNAi flies and the mCherry RNAi control flies. Each dot represents the fluorescence measured in a single fly. Three independent experiments were performed. ${ }^{*} \mathrm{P}<0.05$. Median $\pm \mathrm{SEM}$ are indicated. NS: not significant.

found that this was the case for most tested RNAi lines, Med4, Med9, Med10, Med18, and CDK8 excepted (Table 1). We thus directly measured the efficiency of most of the RNAi lines that did not pass this test by RTqPCR (not shown) and digital RTqPCR (Figure S2). Med18 excepted, the tested lines displayed a strong decrease of the targeted transcripts, suggesting that the corresponding Mediator subunits (Med4, 
Med9, and Med10) may not play an essential role during development.

We have performed survival analysis on 30 RNAi lines after A. fumigatus challenge by expressing the RNAi transgene only at the adult stage. Eight lines yielded a lethal phenotype, that is, uninfected flies succumbed at the same rate as challenged flies (Table 2, Figure 5A). Two lines, Med21 and Med27, displayed a heightened sensitivity to the control injection of PBS, indicating that they are highly-wound sensitive (Table 2, Figure 5B). In both cases, it is thus not possible to assess whether these subunits are specifically involved in host defense. We did not find any enhanced sensitivity to fungal infections for 12 lines (Table 2, Figure 5C). Thus, 10 subunits (Med4, Med9, Med10, Med19, Med20, Med23, Med 24, Med25, Med26, and CycC) do not appear to play an essential role in host defense against $A$. fumigatus since the corresponding RNAi transgene is clearly functional. Finally, seven lines displayed a Med31-like phenotype, although a significant proportion of PBS-injected controls succumbed in the case of Med 11 (Table 2, Figure 5D, Figure S3).

\section{The Med17 Subunit RNAi Mutants Display a Sensitivity Only to Aspergillus fumigatus and Enterococcus faecalis Infections}

Among the lines that shared with the Med31 RNAi line a sensitivity phenotype to A. fumigatus, the Med17 RNAi KD line is of special interest since it targets the expression of Med17, a Mediator complex subunit that has been shown to bind to DIF in vitro and to be required for the Toll-dependent induction of Drosomycin expression in cell culture (25). A similar phenotype was observed with two further independent RNAi lines (Figures S1D, E) and all three KD lines nearly abolished Med17
TABLE 2 | Other Med subunits in host defense against $A$. fumigatus.

\begin{tabular}{lr}
\hline Phenotype & MED subunits \\
\hline $\begin{array}{l}\text { With } \\
\text { phenotype }\end{array}$ & Med 6, Med 8, Med 11, Med14, Med17, Med22, Med30 \\
$\begin{array}{l}\text { Lethal } \\
\text { Wound } \\
\text { sensitive } \\
\begin{array}{l}\text { No } \\
\text { phenotype }\end{array}\end{array}$ \\
\end{tabular}

The subunits indicated in italics may not be efficiently silenced by RNAi and the absence of a phenotype may just reflect this technical problem, thereby preventing a solid conclusion to be drawn.

expression (Figure S1F). It was therefore interesting to determine whether the Med17 RNAi line displayed the same palette of sensitivity to specific microbial infections as the Med31 one. Whereas the Med17 KD line indeed displayed an increased sensitivity to E. faecalis infections in all six performed experiments (Figure 6A), it however was as resistant as control flies to injected $M$. robertsii conidia in the six survival experiments (Figure S4A), unlike the Med31 RNAi line. Finally, the Med17 RNAi line was not sensitive to C. glabrata in five out of six experiments and not susceptible to Ecc15 in six experiments (Figures S4B, C). Thus, even though Med31 and Med17 bind to each other in the Mediator complex, their disruption leads to related but not identical phenotypes. Interestingly, whereas Drosomycin induction by a M. luteus challenge was reduced in the Med17 KD line as expected, it did not affect the induction of BomS1/DIM1 transcripts (Figures 5E, F), even though the BomS1 gene contains a canonical DIF binding site in its promoter (33). Also of note, the expression of some other AMP genes by an E. coli challenge, namely Drosocin

TABLE 1 | Validation of Med RNAi flies lethality at $29^{\circ} \mathrm{C}$.

\begin{tabular}{|c|c|c|c|c|c|}
\hline RNAi strain & Pupae & Hatching flies (\%) & RNAi strain & Pupae & Hatching flies (\%) \\
\hline mCherry & 51 & $51(100 \%)$ & Med19 & 27 & 0 \\
\hline Med1 & 0 & 0 & Med20 & 20 & 0 \\
\hline \#Med4 & 30 & $\begin{array}{c}25 \text { (83.33\%) } \\
10 \text { (33.33\% homozygous) } \\
15 \text { (50\% heterozygous) }\end{array}$ & Med21 & 0 & 0 \\
\hline Med6 & 0 & 0 & Med22 & 0 & 0 \\
\hline Med7 & 0 & 0 & \#Med23 & 0 & 0 \\
\hline Med8 & 0 & 0 & Med24 & 0 & 0 \\
\hline Med9 & 4 & $4(100 \%)$ & Med25 & 0 & 0 \\
\hline \#Med10 & 12 & $\begin{array}{c}100 \% \\
4 \text { (33.33\% homozygous) } \\
8 \text { (66.67\% heterozygous) }\end{array}$ & Med26 & 0 & 0 \\
\hline Med11 & 0 & 0 & Med27 & 0 & 0 \\
\hline Med12 & 0 & 0 & Med28 & 0 & 0 \\
\hline Med13 & 0 & 0 & Med29 & 0 & 0 \\
\hline Med14 & 0 & 0 & \#Med30 & 0 & 0 \\
\hline Med15 & 0 & 0 & Med31 & 0 & 0 \\
\hline Med16 & 8 & 0 & Cyс C & 14 & 0 \\
\hline Med17 & 0 & 0 & CDK8 & 14 & 12 (85.71\%) \\
\hline Med18 & 12 & 10 (83.3\%) & & & \\
\hline
\end{tabular}

Flies were crossed with Ubi-Gal4, tub-Gal80 ts virgins (two males and four females per tube) at $29^{\circ} \mathrm{C}$, and parents were discarded five days post crossing. The offspring was raised at $29^{\circ} \mathrm{C}$ and their viability was assessed.

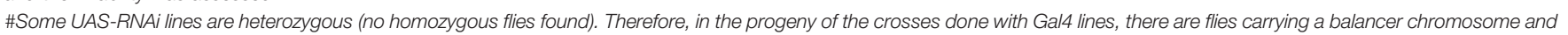
not the UAS-RNAi transgene: these can be identified only at the adult stage, thanks to their genetic markers. 
A

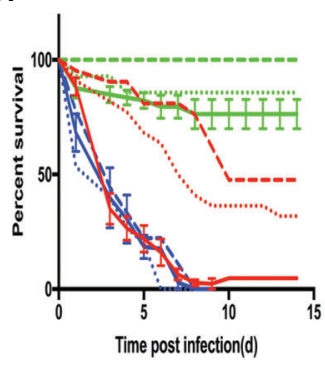

C

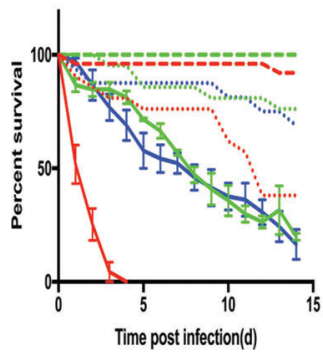

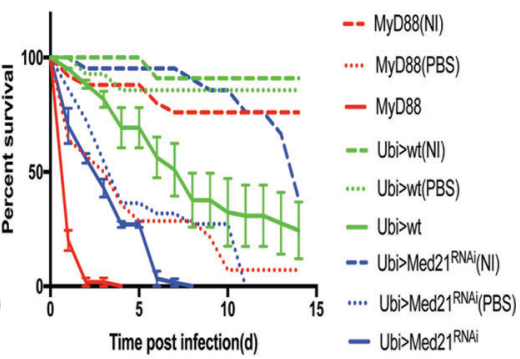

D

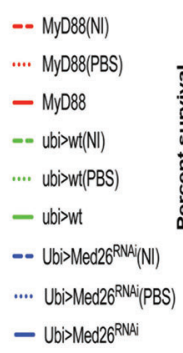

-- MyD88(NI)

... MyD88(PBS)

- MyD88

-- Ubirwt(NI)

.... Ubi>wt(PBS)

- Ubiswt

-- Ubi>Med22 ${ }^{\text {RNAi(NI) }}$

... Ubi>Med22 ${ }^{\text {RNAi}}$ (PBS)

- Ubi>Med22 ${ }^{2 N A i}$
E

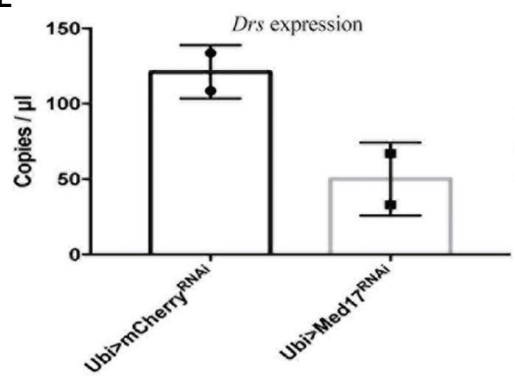

$\mathbf{F}$

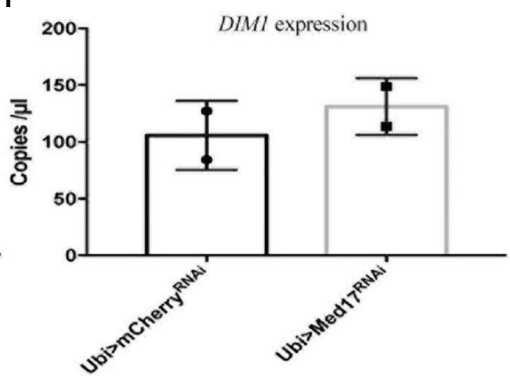

FIGURE 5 | The distinct survival phenotypic categories for other Mediator complex subunits RNAi flies challenged with $A$. fumigatus. Survival of RNAi lines targeting genes encoding other Mediator complex subunits after A. fumigatus infection. MyD88 represents the positive control line (red), Ubi>mCherry RNAi line the wild type control line (green), and Ubi> RNAi line of Mediator complex subunits is shown as a blue line. Each infected line has a non-infected (NI, dashed lines) and a PBSinjected control (dotted lines). (A) Example of a lethal phenotype, uninfected Med15 RNAi flies succumbed at the same rate as challenged flies. (B) Example of a wound-sensitive phenotype, Med21 RNAi flies were sensitive to the injection of PBS. (C) Example of an absence of sensitivity phenotype, Med26 RNAi flies did not show any enhanced sensitivity to infections. (D) Example of sensitivity to A. fumigatus infection. Med17 RNAi flies displayed a Med31-like phenotype. Infected Med17 RNAi flies vs. infected mCherry flies: log-rank test, ${ }^{\star \star \star \star} \mathrm{P}<0.001$. (E, F) Expression level of Drosomycin (E) and DIM1 (F) at $48 \mathrm{~h}$ post $A$. fumigatus infection measured by digital RTqPCR. Each dot represents one sample containing five flies. Mean \pm SEM are indicated.

and CecropinA but not Diptericin, appeared to be upregulated in the Med17 RNAi line (Figures S4 D-G).

\section{Role of Other Med Subunits in Host Defense Against Additional Pathogens}

We next tested the susceptibility of other available RNAi lines against other Med subunit-encoding genes to E. faecalis and found that six of them displayed an enhanced sensitivity to this pathogen (Table 3, Figure S5). We had initially found that Med12, Med15, and Med28 RNAi flies succumbed at the same rate whether challenged or not in two experiments with $A$. fumigatus. In the case of $E$. faecalis infection, the PBS-injected control did not die as rapidly as previously, whereas the bacteriachallenged ones succumbed much faster (Figure S5). Indeed, the
E. faecalis burden was higher than in controls for the Med 15 and Med28 RNAi flies, indicating that these lines are indeed susceptible to $E$. faecalis and not solely to the injection procedure. The important observation is that the Med6 and Med11 lines displayed a sensitivity phenotype after A. fumigatus but not after $E$. faecalis infection.

We further challenged these Mediator subunit RNAi lines with C. glabrata or Ecc15. Most of them tested negative except for Med30 in the case of the pathogenic yeast (Figure 6B) and Med19 for Ecc15 that displayed intermediate sensitivity to these pathogens (Figure 6C, Tables 3, 4).

Finally, we used the $M$. robertsii natural infection model to characterize the Mediator subunit subset and found that Med7, Med27, and Med29 RNAi displayed an enhanced sensitivity to 

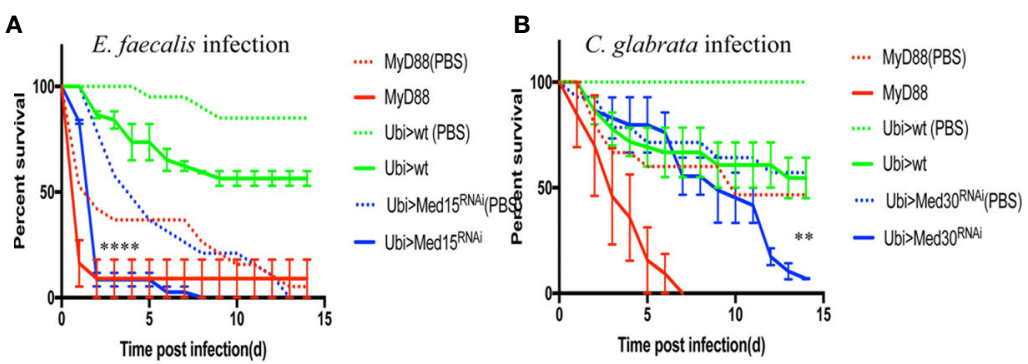

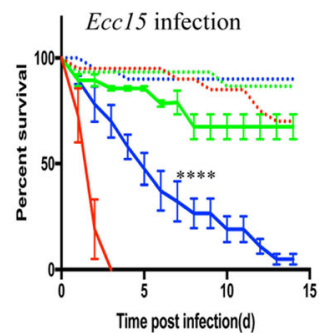

E

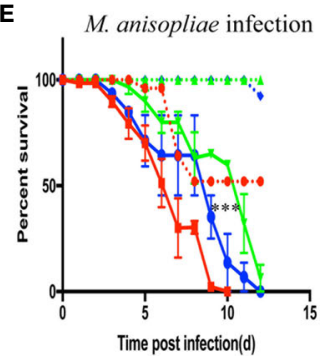

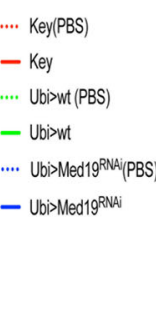

D

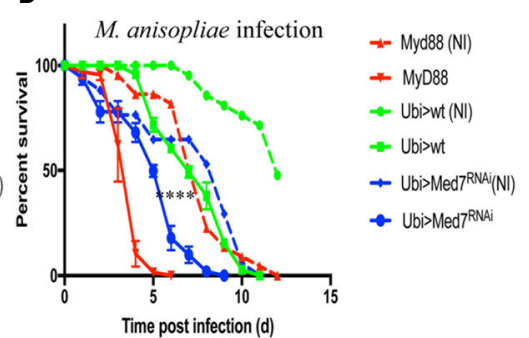

$\mathbf{F}$

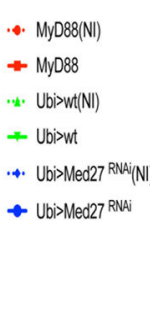

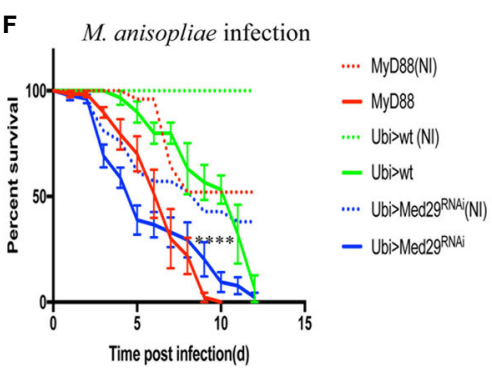

FIGURE 6 | Survival of RNAi flies targeting different Mediator complex subunits after challenge with various pathogens. Survival of other Mediator complex subunits RNAi lines after challenges with either E. faecalis, C. glabrata, ECc15, or M. robertsii. MyD88 was used as positive control line (red) for M. robertsii, C. glabrata or E. faecalis infection, and key was the positive control line (red) for lines infected with Ecc15. Ubi>mCherry RNAi line was the wild type control line (green) and Ubi> RNAi line of Mediator complex subunits is displayed in blue. (A) Survival curves of Med17 RNAi flies, which were susceptible to E. faecalis infection. Infected Med17 RNAi flies vs. infected $m$ Cherry RNAi flies: log-rank test, ${ }^{* \star * \star} \mathrm{P}<0.0001$. The survival curves are representative of six experiments. (B) Med30 RNAi flies were susceptible to C. glabrata infection. Infected Med30 RNAi flies vs. infected $m$ Cherry RNAi flies: log-rank test, ${ }^{* \star} P<0.01$. The survival curves are representative of two experiments. (C) Med19 RNAi flies were susceptible to Ecc15 infection. Infected Med19 RNAi flies vs. infected $m$ Cherry RNAi flies: log-rank test, ${ }^{\star \star \star \star *}$ < 0.0001 . The survival curves are representative of two experiments. (D-F) Med7, Med27, and Med29 RNAi displayed an enhanced sensitivity to M. robertsii "natural" infection. Infected Med7 RNAi flies vs. infected mCherry RNAi flies: log-rank test, ${ }^{\star \star \star \star}{ }^{2} \mathrm{P}<0.0001$. The survival curves are representative of three experiments. Infected Med27 RNAi flies vs. infected $m$ Cherry RNAi flies: log-rank test, ${ }^{* * *} P<0.001$. The survival curves are representative of two experiments. Infected Med29 RNAi flies vs. infected $m$ Cherry flies: log-rank test, ${ }^{* \star \star \star} \mathrm{P}<0.0001$. The survival curves are representative of two experiments. Please, note that the mock-infected flies for Med 7 and Med29 succumbed also rapidly to the procedure, making it difficult to conclude unambiguously on the role of these two subunits in the host defense against M. robertsii "natural" infection. Error bars are SEM.

this challenge although it should be noted that mock-infected Med7 and Med29 RNAi flies also succumbed during the course of this experiment, albeit at a somewhat slower rate (Figures 6DF). This observation for Med 7 and Med 29 is in keeping with the lethality observed in the infections series with A. fumigatus (Table 4). Whereas Med27 is clearly required for host defense against a natural $M$. robertsii infection, we cannot determine unambiguously whether it is also required in the host defense against $A$. fumigatus given its sensitivity to the wound.

\section{DISCUSSION}

We have tested the roles of almost all the subunits of Mediator complex in host defense against infectious pathogens including a filamentous fungus (A. fumigatus), an entomopathogenic fungus in its filamentous form (M. robertsii natural infection; Wang et al., in preparation), or under the hyphal body form (M. robertsii conidia injection; Wang et al., in preparation), yeast (C. glabrata), a Gram-positive bacterium (E. faecalis), and a Gram-negative 
TABLE 3 | Other Med subunits in host defense against other pathogens.

\begin{tabular}{lc}
\hline Pathogens & Other MED subunits \\
\hline E. faecalis & Med 8, Med12?, Med15, Med17, Med22, Med28, Med30 \\
Ecc15 & Med 19 \\
C. glabrata & Med 30 \\
M. robertsii & Med7?, Med27, Med29? \\
Natural infection & \\
\hline
\end{tabular}

The question marks indicate that a sensitivity to the infection procedure may significantly contribute to the phenotype.

bacterium (Ecc15). All of the above-listed pathogens, except for Ecc15, are controlled, at least to some extent, by the Toll pathway. Our results showed that different Mediator subunits displayed distinct sensitivity to infections (Figure 7, Figure S6, Table 4) and reveal differential modes of actions of the Toll pathway.

A recent structural and genetic analysis of the Mediator complex in Mammals has revealed that 15 subunits are essential for cell viability in three human cell types (Table 4), mostly subunits of the core module (except for Med20 in the head module and Med1, Med9, Med19, Med26 in the middle module) and three tail subunits interfacing with the head and middle modules, namely Med27, Med28, and Med30 as well as the scaffolding subunit Med14. These essential subunits appear to be indispensable for the function of the Mediator complex in globally recruiting RNA Polymerase B to promoters to form the preinitiation complex (36). Here, we find that most subunits are required during development, except for Med4, Med9, Med10, Med18, Med19, Med20, CycC, and CDK8 for which some escaper pupae or adults were obtained (Table 1). It is likely that the Med18 phenotype is due to a partial attenuation of its expression at the mRNA level as determined by regular and digital RTqPCR. We have not checked the efficiency of RNA interference for $\operatorname{Med16}, \operatorname{Med19}, \mathrm{CycC}$, and $C D K 8$, and thus cannot formally exclude a similar explanation for these subunits. In contrast, the RNAi approach seems to be efficient as regards Med4, Med9, Med10, and Med20. While Med9 and Med20 are also not indispensable in Mammals, Med4 and Med10 may in contrast be essential in Mammals but not in insects, although it is difficult to compare viability at the cellular $v s$. at the organismal level. Indeed, the depletion of Med subunits only at the adult stage led to the demise of eight uninfected RNAi mutant lines in the experiments in which susceptibility to A. fumigatus was tested. This occurred also when subunits not homologous to essential mammalian subunits were targeted, i.e.,

TABLE 4 | Summary of Med subunits in host defense against pathogens.

A. fumigatus E. faecalis M. robertsii natural infection C. glabrata Ecc15 M. robertsii Injection Lethality at $29^{\circ} \mathrm{C}$ RNAi efficiency by dPCR

\begin{tabular}{|c|c|c|c|c|c|c|c|c|}
\hline Med1 & $\mathrm{L}$ & - & - & - & - & / & ++ & / \\
\hline Med4 & - & - & - & - & - & / & $+/-$ & + \\
\hline$\overline{\text { Med6 }}$ & + & - & - & - & - & / & ++ & / \\
\hline$\overline{\text { Med7 }}$ & $\mathrm{L}$ & - & $+?$ & - & - & / & ++ & / \\
\hline$\overline{\text { Med8 }}$ & + & + & - & - & - & / & ++ & / \\
\hline$\overline{\text { Med9 }}$ & - & - & - & - & - & / & $+/-$ & + \\
\hline Med10 & - & - & - & - & - & / & $+/-$ & + \\
\hline Med11 & + & - & - & - & - & / & ++ & / \\
\hline$\overline{\text { Med12 }}$ & $\mathrm{L}$ & + & / & - & - & / & ++ & / \\
\hline Med13 & $\mathrm{L}$ & - & - & - & - & / & ++ & / \\
\hline Med14 & + & / & - & - & / & / & ++ & / \\
\hline$\overline{\text { Med15 }}$ & $\mathrm{L}$ & + & - & - & - & / & ++ & / \\
\hline Med16 & $\mathrm{L}$ & - & - & - & - & / & + & / \\
\hline Med17 & + & + & - & - & - & - & ++ & + \\
\hline Med18 & - & - & - & - & - & / & $+/-$ & $+/-$ \\
\hline$\overline{\text { Med19 }}$ & - & - & - & - & + & / & + & / \\
\hline Med20 & - & - & - & - & - & / & + & + \\
\hline Med21 & $-?$ & - & / & - & / & / & ++ & / \\
\hline Med22 & + & + & - & - & - & / & ++ & / \\
\hline$\overline{\text { Med23 }}$ & - & - & - & - & - & / & ++ & / \\
\hline Med24 & - & - & - & - & - & / & ++ & / \\
\hline Med25 & - & - & - & - & - & / & ++ & / \\
\hline Med26 & - & - & - & - & - & / & ++ & / \\
\hline Med27 & $-?$ & - & + & - & - & / & ++ & / \\
\hline$\overline{\text { Med28 }}$ & $\mathrm{L}$ & + & - & - & - & / & ++ & / \\
\hline Med29 & $\mathrm{L}$ & - & $+?$ & - & - & / & ++ & / \\
\hline Med30 & + & + & - & + & - & / & ++ & / \\
\hline$\overline{\text { Med31 }}$ & + & + & - & - & - & + & ++ & + \\
\hline Cус C & - & - & - & - & - & / & + & / \\
\hline CDK8 & - & - & - & - & - & / & $+/-$ & / \\
\hline
\end{tabular}

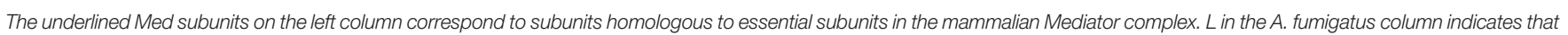

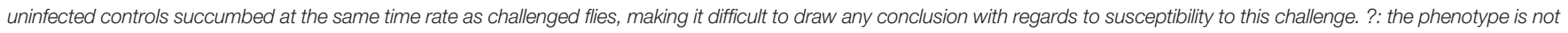

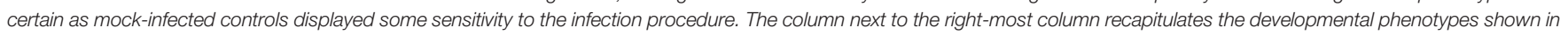

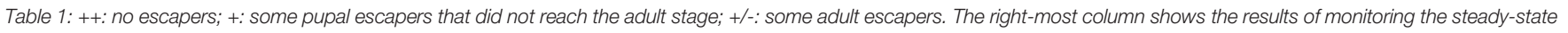

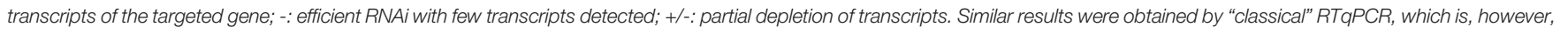
not as precise. /: not tested. Med subunit in bold: the RNAi line is unlikely to function efficiently. 


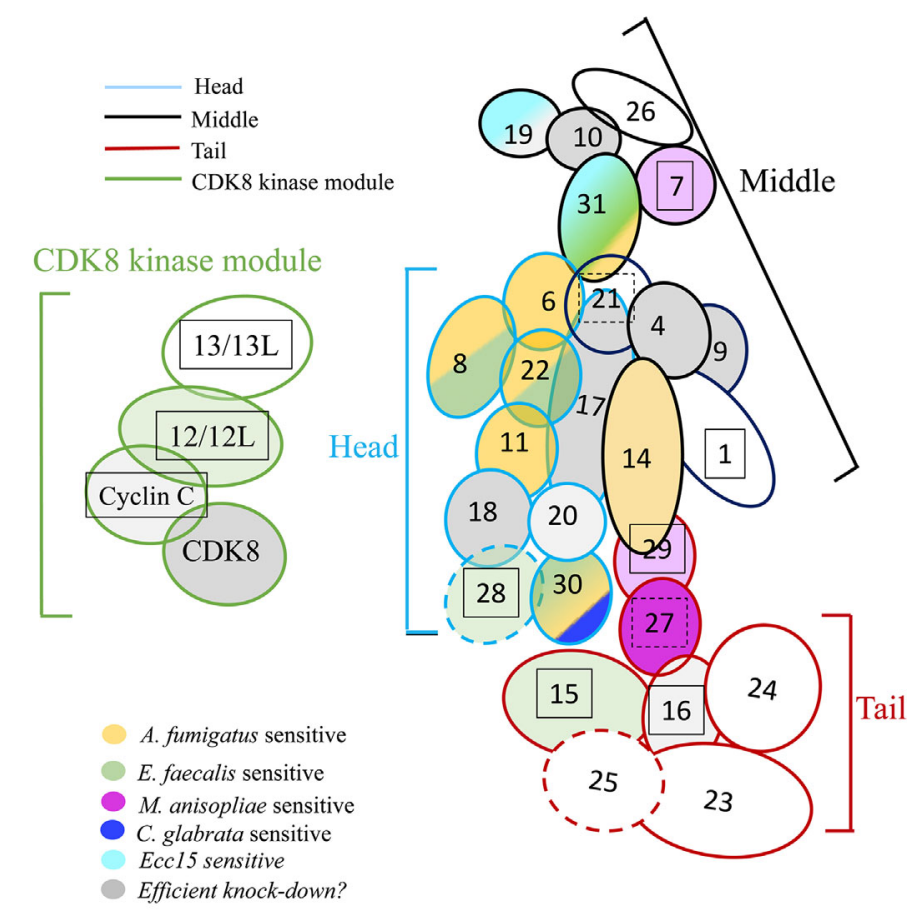

FIGURE 7 | Roles of Mediator complex modules against infectious pathogens. The Mediator complex is composed of a central complex and of the CDK kinase module (CKM). The central complex has three modules: the head (light blue circumference), the middle (black circumference), and the tail (red circumference). Med14 constitutes a scaffolding subunit indicated by dark blue. The CKM (green circumference) consists of four subunits: CDK8 (or its paralog CDK19), Med12 (or its paralog Med12L), Med13 (or its paralog Med13L), and Cyclin C. Subunits sensitive to A. fumigatus are shown in yellow; subunits sensitive to E. faecalis in green, light green when the phenotype is uncertain due to a lethality observed in the A. fumigatus experiments (Med12); subunits sensitive to $M$. robertsii natural infection are displayed in dark purple, light purple when the phenotype is uncertain; subunits sensitive to M. robertsii injection are shown in orange (only Med17 and Med31 RNAi flies have been tested); subunits sensitive to C. glabrata infection are pictured in dark blue whereas subunits sensitive to Ecc15 infection are light blue. Lines for which the RNAi may not be effective are displayed in gray; a lighter gray indicates lines in which pupae developed upon continuous expression of the RNAi transgene during development, yet did not yield any adult flies (metamorphosis phenotype). Lines that yielded a lethal phenotype in the uninfected controls in the A. fumigatus experiments when gene expression was inhibited in the adult display a boxed subunit number, which is dashed when sensitivity was observed in the wounding (PBS-injected) but not the uninfected controls.

Med1, Med12, Med13, Med15, Med16, Med28, and Med29. It was unexpected that the uninfected controls for the same lines did not reveal any lethality when E. faecalis was tested later in another series of survival experiments (Table 4). This uninfected control was not performed for survival experiments with other tested pathogens; however, mock-infected controls were performed in these experiments and did not reveal any unusual viability issue when kept at $29^{\circ} \mathrm{C}$, except where indicated in Table 4 ( $M$. robertsii natural infections). We suspect that conditions were slightly harsher in the A. fumigatus survival experiment series, resulting in a more efficient RNAi and depletion of the cognate subunit thus revealing their essential function, even in the context of the adult in which cells do not divide, somatic and germinal stem cells excepted. In contrast to classical mutations induced by chemical mutagenesis or CRISPR-Cas9, a fundamental difference with conditional RNAi is that the proteins are made in the cell prior to the induction of the RNAi. Even if the interference is $100 \%$ effective, the limiting factor to express the phenotype will be the relative stability of the protein already made, which may also vary from one target to the other, thus modulating the gamut of observable phenotypes. Indeed, it has already been noted in genome-wide genetic screens that the genes identified by RNA interference and those retrieved with random mutagenesis techniques differ extensively (37). Thus, this limitation has to be kept in mind when interpreting the results from this study.

One interesting observation relates to the CDK8 module: the Med12 and Med13 subunits appear to be essential in the adult, but not $\mathrm{CycC}$ and $\mathrm{CDK} 8$, even though the RNAi targeting each of the latter two subunits is effective, at least for $C y c C$ since no adults were retrieved when the RNAi transgene was expressed continuously throughout development. One possibility would be that the $\mathrm{CycC}$ and $\mathrm{CDK} 8$ proteins are more stable, although one should note that the survival experiments were monitored for over two weeks while the whole development at $29^{\circ} \mathrm{C}$ occurs in less than ten days. An alternative is that Med12 and Med13 have differing functions from the $\mathrm{Cdk} 8 / \mathrm{CycC}$ kinase module of the Mediator complex, in keeping with a previous study (38).

We found that mutations affecting some of the Mediator complex subunits were not sensitive to any pathogen we have 
tested: Med4, Med9, Med10, Med20, Med26 of the core module, Med23, Med24, Med 25 of the tail module, and Med13 and CycC for the CDK8 kinase module (Figure 7, Figure S6, Table 4). Most of these subunits correspond to nonessential subunits in the mammalian complex, except for Med4 and Med10. Med20 is the only subunit of the head module not playing a role in the host defense against the pathogens we have tested and consistently is also the only subunit of the head not essential in the mammalian complex (36).

The only RNAi lines displaying a sensitivity to A. fumigatus infection affect the expression of genes encoding subunits of the head module with two exceptions, Med31 in the middle module and Med30 in the tail module (Figure 7, Figure S6, Tables 2, 4). With respect to $E$. faecalis infection, the RNAi susceptible lines correspond to many subunits of the head module (Med8, Med17, Med22, Med28). We cannot formally exclude that Med6 and Med11 would also have tested positive had the conditions been as stringent as for the A. fumigatus infections. Beyond the head module, Med15, Med28, and Med30 in the tail module and Med31 in the middle module also displayed an E. faecalis infection sensitivity phenotype (Figure 7, Figure S6, Tables 3, 4). As the Med15 and Med28 RNAi lines uninfected flies displayed a lethal phenotype in the A. fumigatus survival experiment series, we cannot exclude that these subunits may also be required for host defense against this fungus. It follows that it is an open possibility that host defense against these two pathogens involves the same set of Mediator subunits (Figure 7, Table 4).

Med30 expression down-regulation was the only one that led to a sensitivity to C. glabrata infection and corresponds to a subunit of the tail module of Mediator also required for host defense against A. fumigatus and E. faecalis (Figure 7, Figure S6, Tables 3, 4). This finding suggests that host defense against C. glabrata is strikingly distinct from that of the two microbes discussed so far. Even though DIF plays a central role in host defense against some fungal and Gram-positive bacterial infections, it is not required in the host defense against C. glabrata and its involvement in the defense against $A$. fumigatus has not been reported yet $(6,28,39)$.

Only the Med19 RNAi line displayed sensitivity to Ecc15 infection (Figure 7, Figure S6, Tables 3, 4). It is not clear at this stage whether this phenotype reflects an impaired function of the IMD pathway in the case of Med19. We expected to find a role for Med25 as it has been identified as being required for IMD pathway activation in an RNAi screen performed on cultured cells (40). This observation suggests that the regulation of the IMD pathway in cultured cells and in vivo may not be identical, at least as regards the role of the Mediator complex.

Med30 and Med31 display unique phenotypes, in that they are required for host defense against three types of infections, including E. faecalis and A. fumigatus. Med30 RNAi flies are the only ones to be sensitive to C. glabrata whereas Med31 flies are susceptible to injected $M$. robertsii conidia but not in a natural infection paradigm. Unexpectedly, distinct subunits appear to be involved in the response to this type of infection, in which the pathogen breaks through the cuticle, namely Med27 and possibly Med7 and Med29. This result is striking in that the Toll pathway and DIF are both required in the host defense against $M$. robertsii in either infection model (4, Wang et al., in preparation). Future work will tell whether the subunits required for host defense against $M$. robertsii in the natural infection model are required locally, for instance in the hypodermis or whether they mediate the action of DIF in the fat body. Of note, only two subunits, Med31 and Med17, have been tested in the M. robertsii conidia injection model (Table 4, Figure S6).

Med17 is the subunit shown to bind directly to DIF in vitro (25). DIF is required for the induction of multiple genes regulated by the Toll pathway (41), including Drosomycin and BomS1/DIM1. It was therefore surprising to observe that the induced BomS1 expression was not impaired in Med17 RNAi KD flies, although that of Drosomycin was affected. This situation is reminiscent of that documented for Med1 during Drosophila development: it recruits GATA transcription factors only for a subset of genes regulated by these factors (24). Thus, it is likely that the context plays an essential role, not only with respect to the regulation of specific subsets of genes through a given set of Mediator subunits, but also depending on the pathogen and infection route. Indeed, there is only a limited overlap of genes with an altered expression in the natural infection or conidia injection models of $M$. robertsii injection. It should also be kept in mind that host defense is not limited to resistance, for instance through AMPs, but involves the dimension of resilience/ tolerance whereby the host withstands or repairs damages exerted by pathogen virulence factors or its own immune response (42-44). As exemplified by the finding that the Caenorhabditis elegans Med15 is required for host defense against Pseudomonas aeruginosa and detoxification of some of the toxins it secretes such as phenazines (45), it will be therefore interesting to determine whether some of the Med subunits identified in this study for their involvement in host defense actually mediate resistance, resilience, or both.

\section{DATA AVAILABILITY STATEMENT}

The datasets generated for this study are available on request to the corresponding author.

\section{AUTHOR CONTRIBUTIONS}

$\mathrm{CH}$ performed most experiments described in this work and participated in the genetic screen that led to this work. RX developed the A. fumigatus infection model and started the genetic screen that led to the identification of Med subunit genes; he also characterized the Med17 RNAi phenotype and performed the digital PCR experiments. SL and ZL provided guidance and supervision for the experiments. DC and DF played a key role in designing the genetic screen, and DC organized the actual implementation of the genetic screen and coordinated the logistics. DF and $\mathrm{CH}$ designed the experiments, analyzed the data, and wrote the manuscript. All authors contributed to the article and approved the submitted version. 


\section{FUNDING}

This work was funded through the Incubation Project for Innovation Team of the Guangzhou Medical University (\# B1850004105) and the 111 Project (\# D18010) as well as support from the China 1,000 Talent program to DF.

\section{ACKNOWLEDGMENTS}

We thank Guiying Lin, Xiaomin Chang, Jin Li, Jianmei Li, Xinzhu Chen, Yuqi Yang, Jianglong Guo, and Xuejun Hong for the help in some of the experiments and for the genetic screen. The expert technical contribution of Luhong Lin, Yongxin Liao, Fengyi Zhang, Yanzhen He for the screen is gratefully acknowledged. We are

\section{REFERENCES}

1. Sugui JA, Kwon-Chung KJ, Juvvadi PR, Latgé J-P, Steinbach WJ. Aspergillus fumigatus and Related Species. Cold Spring Harbor Perspect Med (2015) 5(2): a019786. doi: 10.1101/cshperspect.a019786

2. Philippe B, Ibrahim-Granet O, Prevost MC, Gougerot-Pocidalo MA, Sanchez Perez M, Van der Meeren A, et al. Killing of Aspergillus fumigatus by alveolar macrophages is mediated by reactive oxidant intermediates. Infect Immun (2003) 71(6):3034-42. doi: 10.1128/IAI.71.6.3034-3042.2003

3. Buchon N, Silverman N, Cherry S. Immunity in Drosophila melanogasterfrom microbial recognition to whole-organism physiology. Nat Rev Immunol (2014) 14(12):796-810. doi: 10.1038/nri3763

4. Gottar M, Gobert V, Matskevich AA, Reichhart JM, Wang C, Butt TM, et al. Dual Detection of Fungal Infections in Drosophila via Recognition of Glucans and Sensing of Virulence Factors. Cell (2006) 127(7):1425-37. doi: 10.1016/ j.cell.2006.10.046

5. Issa N, Guillaumot N, Lauret E, Matt N, Schaeffer-Reiss C, Van Dorsselaer A, et al. The Circulating Protease Persephone Is an Immune Sensor for Microbial Proteolytic Activities Upstream of the Drosophila Toll Pathway. Mol Cell (2018) 69(4):539-550 e6. doi: 10.1016/j.molcel.2018.01.029

6. Rutschmann S, Jung AC, Hetru C, Reichhart J-M, Hoffmann JA, Ferrandon D. The Rel protein DIF mediates the antifungal, but not the antibacterial, response in Drosophila. Immunity (2000) 12:569-80. doi: 10.1016/S10747613(00)80208-3

7. Manfruelli P, Reichhart JM, Steward R, Hoffmann JA, Lemaitre B. A mosaic analysis in Drosophila fat body cells of the control of antimicrobial peptide genes by the Rel proteins Dorsal and DIF. EMBO J (1999) 18(12):3380-91. doi: 10.1093/emboj/18.12.3380

8. Meng X, Khanuja BS, Ip YT. Toll receptor-mediated Drosophila immune response requires Dif, an NF- kappaB factor. Genes Dev (1999) 13(7):792-7. doi: $10.1101 / \operatorname{gad} \cdot 13.7 .792$

9. Ferrandon D, Imler JL, Hetru C, Hoffmann JA. The Drosophila systemic immune response: sensing and signalling during bacterial and fungal infections. Nat Rev Immunol (2007) 7:862-74. doi: 10.1038/nri2194

10. Clemmons AW, Lindsay SA, Wasserman SA. An effector Peptide family required for Drosophila toll-mediated immunity. PloS Pathog (2015) 11(4): e1004876. doi: 10.1371/journal.ppat.1004876

11. Lindsay SA, Lin SJH, Wasserman SA. Short-Form Bomanins Mediate Humoral Immunity in Drosophila. J Innate Immun (2018) 10(4):306-14. doi: 10.1159/000489831

12. Cohen LB, Lindsay SA, Xu Y, Lin SJH, Wasserman SA. The Daisho Peptides Mediate Drosophila Defense Against a Subset of Filamentous Fungi. Front Immunol (2020) 11:9. doi: 10.3389/fimmu.2020.00009

13. Lemaitre B, Nicolas E, Michaut L, Reichhart JM, Hoffmann JA. The dorsoventral regulatory gene cassette spätzle/Toll/cactus controls the potent antifungal response in Drosophila adults. Cell (1996) 86:973-83. doi: 10.1016/ S0092-8674(00)80172-5 indebted to Prof. Ni and his collaborators (Tsinghua University) for giving us access to his resource of Trip RNAi lines (Tsinghua Fly Center) and also the Vienna Drosophila Resource Center (VDRC, www.vdrc.at) and Bloomington Drosophila Stock Center (NIH P40OD018537) for their resource. We thank Anne Beauvais and Jean-Paul Latge for the gift of the RFP-labeled A. fumigatus wild-type strain, Erjun Ling for the gift of the PPO1 antibody. We thank Dr. Kwang-Zin Lee for critical reading of the manuscript.

\section{SUPPLEMENTARY MATERIAL}

The Supplementary Material for this article can be found online at: https://www.frontiersin.org/articles/10.3389/fimmu.2020.478958/ full\#supplementary-material

14. Ni JQ, Zhou R, Czech B, Liu LP, Holderbaum L, Yang-Zhou D, et al. A genome-scale shRNA resource for transgenic RNAi in Drosophila. Nat Methods (2011) 8(5):405-7. doi: 10.1038/nmeth.1592

15. Dietzl G, Chen D, Schnorrer F, Su KC, Barinova Y, Fellner M, et al. A genomewide transgenic RNAi library for conditional gene inactivation in Drosophila Nature (2007) 448(7150):151-6. doi: 10.1038/nature05954

16. McGuire SE, Mao Z, Davis RL. Spatiotemporal gene expression targeting with the TARGET and gene-switch systems in Drosophila. Sci STKE (2004) 2004 (220):pl6. doi: 10.1126/stke.2202004pl6

17. Thompson CM, Young RA. General requirement for RNA polymerase II holoenzymes in vivo. Proc Natl Acad Sci USA (1995) 92p(10):4587-90. doi: 10.1073/pnas.92.10.4587

18. Kim YJ, Bjorklund S, Li Y, Sayre MH, Kornberg RD. A multiprotein mediator of transcriptional activation and its interaction with the C-terminal repeat domain of RNA polymerase II. Cell (1994) 77(4):599-608. doi: 10.1016/00928674(94)90221-6

19. Bourbon HM. Comparative genomics supports a deep evolutionary origin for the large, four-module transcriptional mediator complex. Nucleic Acids Res (2008) 36(12):3993-4008. doi: 10.1093/nar/gkn349

20. Soutourina J. Transcription regulation by the Mediator complex. Nat Rev Mol Cell Biol (2018) 19(4):262-74. doi: 10.1038/nrm.2017.115

21. Poss ZC, Ebmeier CC, Taatjes DJ. The Mediator complex and transcription regulation. Crit Rev Biochem Mol Biol (2013) 48(6):575-608. doi: 10.3109/ 10409238.2013.840259

22. Yin JW, Wang G. The Mediator complex: a master coordinator of transcription and cell lineage development. Development (2014) 141 (5):977-87. doi: 10.1242/dev.098392

23. Bosveld F, van Hoek S, Sibon OC. Establishment of cell fate during early Drosophila embryogenesis requires transcriptional Mediator subunit dMED31. Dev Biol (2008) 313(2):802-13. doi: 10.1016/j.ydbio. 2007.11.019

24. Immarigeon C, Bernat-Fabre S, Auge B, Faucher C, Gobert V, Haenlin M, et al. Drosophila Mediator Subunit Med1 Is Required for GATA-Dependent Developmental Processes: Divergent Binding Interfaces for Conserved Coactivator Functions. Mol Cell Biol (2019) 39(7):e00477-18. doi: 10.1128/ MCB.00477-18

25. Park JM, Kim JM, Kim LK, Kim SN, Kim-Ha J, Kim JH, et al. Signal-induced transcriptional activation by Dif requires the dTRAP80 mediator module. $\mathrm{Mol}$ Cell Biol (2003) 23(4):1358-67. doi: 10.1128/MCB.23.4.1358-1367.2003

26. Guruharsha KG, Rual JF, Zhai B, Mintseris J, Vaidya P, Vaidya N, et al. A protein complex network of Drosophila melanogaster. Cell (2011) 147 (3):690-703. doi: 10.1016/j.cell.2011.08.047

27. Thurmond J, Goodman JL, Strelets VB, Attrill H, Gramates LS, Marygold SJ, et al. FlyBase 2.0: the next generation. Nucleic Acids Res (2019) 47(D1):D75965. doi: 10.1093/nar/gky1003

28. Quintin J, Asmar J, Matskevich AA, Lafarge MC, Ferrandon D. The Drosophila Toll Pathway Controls but Does Not Clear Candida glabrata Infections. J Immunol (2013) 190(6):2818-27. doi: 10.4049/jimmunol.1201861 
29. Madic J, Zocevic A, Senlis V, Fradet E, Andre B, Muller S, et al. Three-color crystal digital PCR. Biomol Detect Quantif (2016) 10:34-46. doi: 10.1016/ j.bdq.2016.10.002

30. Limmer S, Haller S, Drenkard E, Lee J, Yu S, Kocks C, et al. Pseudomonas aeruginosa RhlR is required to neutralize the cellular immune response in a Drosophila melanogaster oral infection model. Proc Natl Acad Sci USA (2011) 108(42):17378-83. doi: 10.1073/pnas.1114907108

31. Li X, Ma M, Liu F, Chen Y, Lu A, Ling QZ, et al. Properties of Drosophila melanogaster prophenoloxidases expressed in Escherichia coli. Dev Comp Immunol (2012) 36(4):648-56. doi: 10.1016/j.dci.2011.11.005

32. Duneau D, Ferdy JB, Revah J, Kondolf H, Ortiz GA, Lazzaro BP, et al. Stochastic variation in the initial phase of bacterial infection predicts the probability of survival in D. melanogaster. Elife (2017) 6:e28298. doi: 10.7554/ eLife. 28298

33. Busse MS, Arnold CP, Towb P, Katrivesis J, Wasserman SA. A kappaB sequence code for pathway-specific innate immune responses. EMBO J (2007) 26(16):3826-35. doi: 10.1038/sj.emboj.7601798

34. Lemaitre B, Reichhart JM, Hoffmann JA. Drosophila host defense : differential display of antimicrobial peptide genes after infection by various classes of microorganisms. Proc Natl Acad Sci USA (1997) 94:14614-9. doi: 10.1073/ pnas.94.26.14614

35. Alarco AM, Marcil A, Chen J, Suter B, Thomas D, Whiteway M. Immunedeficient Drosophila melanogaster: a model for the innate immune response to human fungal pathogens. J Immunol (2004) 172(9):5622-8. doi: 10.4049/ jimmunol.172.9.5622

36. El Khattabi L, Zhao H, Kalchschmidt J, Young N, Jung S, Van Blerkom P, et al. A Pliable Mediator Acts as a Functional Rather Than an Architectural Bridge between Promoters and Enhancers. Cell (2019) 178(5):1145-1158 e20. doi: 10.1016/j.cell.2019.07.011

37. Yamamoto S, Jaiswal M, Charng WL, Gambin T, Karaca E, Mirzaa G, et al. A drosophila genetic resource of mutants to study mechanisms underlying human genetic diseases. Cell (2014) 159(1):200-14. doi: 10.1016/ j.cell.2014.09.002

38. Kuuluvainen E, Hakala H, Havula E, Sahal Estime M, Ramet M, Hietakangas V, et al. Cyclin-dependent kinase 8 module expression profiling reveals requirement of mediator subunits 12 and 13 for transcription of Serpent- dependent innate immunity genes in Drosophila. J Biol Chem (2014) 289 (23):16252-61. doi: 10.1074/jbc.M113.541904

39. Rutschmann S, Kilinc A, Ferrandon D. The Toll pathway is required for resistance to Gram-positive bacterial infections in Drosophila. J Immunol (2002) 168:1542-6. doi: 10.4049/jimmunol.168.4.1542

40. Valanne S, Myllymaki H, Kallio J, Schmid MR, Kleino A, Murumagi A, et al. Genome-wide RNA interference in Drosophila cells identifies G proteincoupled receptor kinase 2 as a conserved regulator of NF-kappaB signaling. J Immunol (2010) 184(11):6188-98. doi: 10.4049/jimmunol.1000261

41. Irving P, Troxler L, Heuer TS, Belvin M, Kopczynski C, Reichhart J, et al. A genome-wide analysis of immune responses in Drosophila. Proc Natl Acad Sci (USA) (2001) 98:15119-24. doi: 10.1073/pnas.261573998

42. Medzhitov R, Schneider DS, Soares MP. Disease tolerance as a defense strategy. Science (2012) 335(6071):936-41. doi: 10.1126/science.1214935

43. Ferrandon D. The complementary facets of epithelial host defenses in the genetic model organism Drosophila melanogaster: from resistance to resilience. Curr Opin Immunol (2013) 25(1):59-70. doi: 10.1016/j.coi.2012.11.008

44. Soares MP, Teixeira L, Moita LF. Disease tolerance and immunity in host protection against infection. Nat Rev Immunol (2017) 17(2):83-96. doi: 10.1038/nri.2016.136

45. Pukkila-Worley R, Feinbaum RL, McEwan DL, Conery AL, Ausubel FM. The evolutionarily conserved mediator subunit MDT-15/MED15 links protective innate immune responses and xenobiotic detoxification. PloS Pathog (2014) 10(5):e1004143. doi: 10.1371/journal.ppat.1004143

Conflict of Interest: The authors declare that the research was conducted in the absence of any commercial or financial relationships that could be construed as a potential conflict of interest.

Copyright (c) 2021 Huang, Xu, Liégeois, Chen, Li and Ferrandon. This is an openaccess article distributed under the terms of the Creative Commons Attribution License (CC BY). The use, distribution or reproduction in other forums is permitted, provided the original author(s) and the copyright owner(s) are credited and that the original publication in this journal is cited, in accordance with accepted academic practice. No use, distribution or reproduction is permitted which does not comply with these terms. 